\title{
What a future talent would say about translation automation?
}

\author{
Elsa Sklavounou ${ }^{1}$ \\ ${ }^{1}$ RWS Senior Director, International Partnerships \\ esklavounoulrws.com
}

\section{Introduction}

This keynote script aims to present future talent's vision on the role translation automation plays in building a continuous intelligent content supply chain under subject matter expert supervision highlighting the importance of the localization experts and professionals.

Artificial intelligence and automation in general are defining corporate digital content strategy. When the automation is enabled thanks to AI-based language technology within localization project workflows, RWS, through the RWS Campus, aims to inspire great futures in localization and to be recognized in our industry for developing localization talent and markets worldwide: more than 200 universities in 37 countries are figuring amongst the academic institutions RWS supports through

- University events (55 universities across 22 countries)

- Advisory programs (8 universities)

- Presentations and Workshops (38 universities in 17 countries)

- Internship Programs (80+ universities in 20+ countries)

Highly skilled interns are getting hired

- for permanent roles at RWS in 17 countries

- for freelance roles at RWS in 8 countries

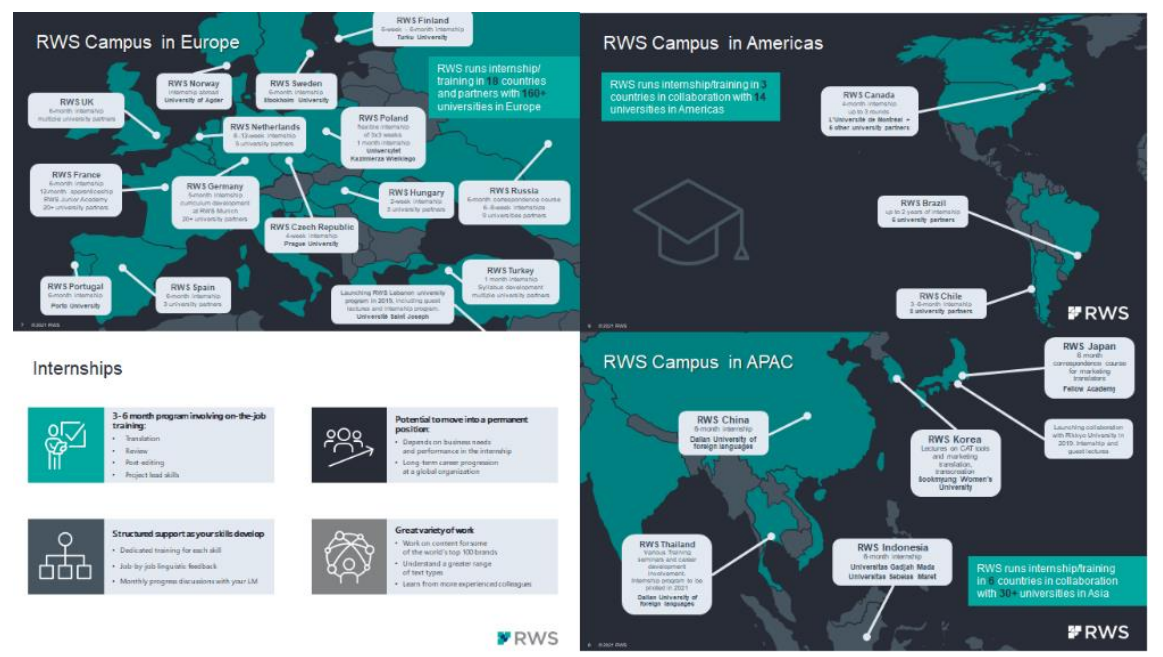

Fig. 1. RWS Campus Internships 


\section{$2 \quad$ Building an intelligent content supply chain}

The priority for any business must be its reputation - without it, it is doomed to fail. Compliance with local laws and regulations is essential, and businesses should have a strategy in place for how they will ensure that their content assets continue to meet ever-changing rules around the world. Automatic translation has a vital role to play in helping to produce Multilingual Content - as does weaving a Corporate Content Fabric - where Artificial Intelligence, based on subject-matter expertise, helps businesses at every stage of their content development - from creation, through translation, to complex delivery.

Building an intelligent content supply chain under an optimized operating model from content creation to multi-channel content distribution requires companies to adopt the right Content Management Solution for their business-critical information so as to enable

- Full Digital Transformation

- Successful AI Implementation

A component-based content infrastructure provides optimal content reuse and life cycle management across any device and any format. By ensuring it integrates seamlessly with existing systems you can achieve unified collaboration for authoring and reviewing, and improved governance at scale in 250+ languages, fully secured and cost-optimized.

Content management in a multilanguage, multichannel world is fiendishly difficult and makes it hard to maximize the value of your data because of these complex content deliveries.

However, there is a way...

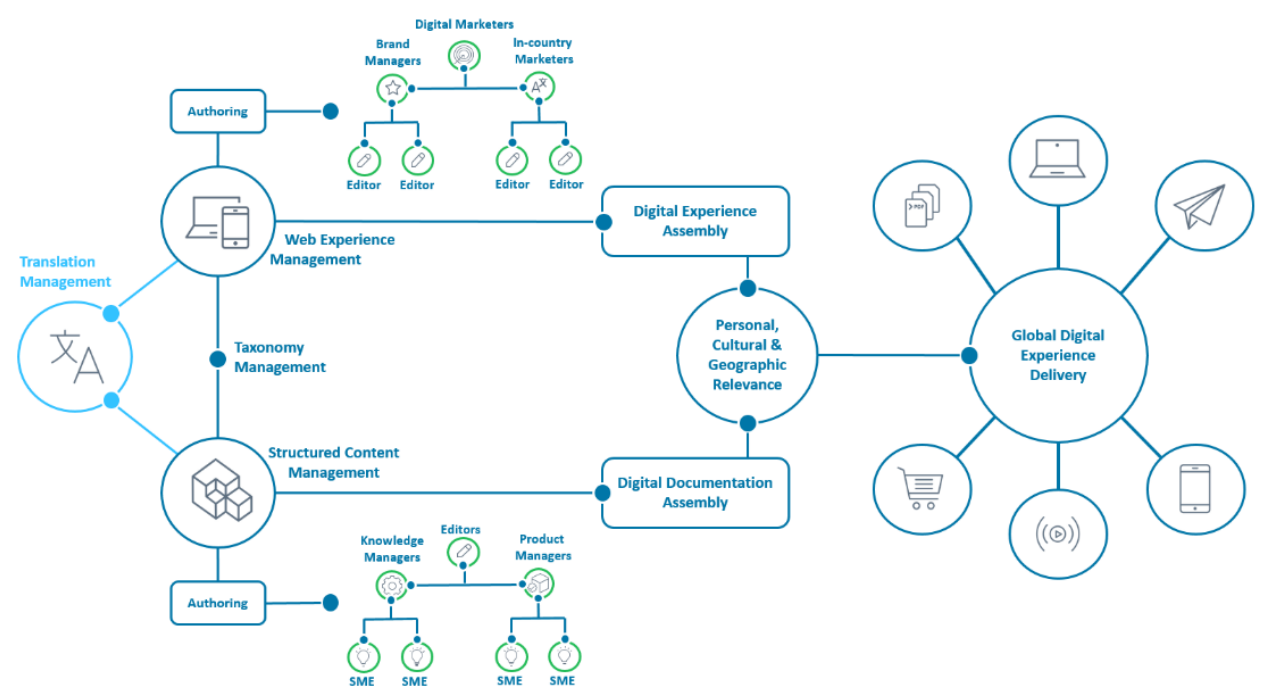

Fig. 2. Intelligent Content Supply Chain 
Intelligent Content platforms allow companies to optimize and automate content processes at scale, so they can cost-efficiently import, create, manage, localize and distribute a wide spectrum of content types - from highly structured regulatory content to engaging marketing content manageable throughout the content life cycle.

\section{Streamlining intelligence from multi-target content}

Single-sourcing of content reduces cost of content development $(30 \%)$ and localization (30\%-50\%). Shifting translation automation from Localization to Authoring and Management flows allows the immersion of locals in the taxonomy model based on the delivery target destinations not only from delivery channel and domain perspective but also it handles local language variants. The advantages in moving the machine translation to the authoring stage of a well-defined content supply chain are multiple:

- Delivering the right post-sale experience

Enhance customer experience by delivering the technical post-sale content experience that matches the personalized, dynamic and rich pre-sale content experience.

- Shorter turnaround times

The advanced version management for the creation, management, review, edit and delivery steps are operated within a single workflow process and the streamlined collaboration contribute to eliminate manual, error-prone reviews and stop being the middleman when reviewers disagree. Empower SMEs to contribute feedback for cross-departmental sharing.

- Better fit of content to the form-factor of the delivery channel

Automated rendering into multiple formats based on style sheets reduces - or completely eliminates Desktop Publishing costs. Eliminate manual post-processing for translated content layout fixes as content is developed at database level and pulled based on the delivery channel depending on the digital experience.

- Ability to train MT based on the delivery channel

By enabling content reuse, breaking publications down into smaller, reusable pieces, managed on a single platform and available to all stakeholders accelerating the digital experience is reducing costs, allows to feed back as many localizations as digital experiences tagged for machine learning purposes, via the respective metadata by the subject matter experts and content developers within a circular workflow for long content life cycle. 


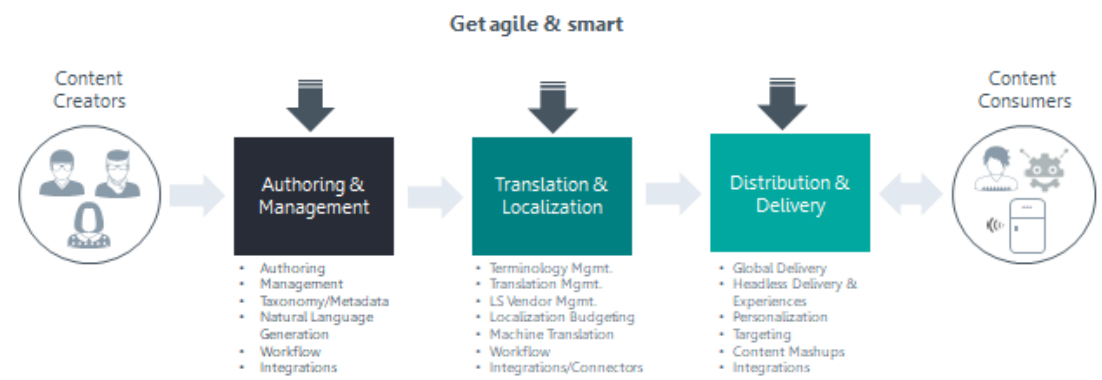

The future RWS talent is here to transform content complexity to intelligence

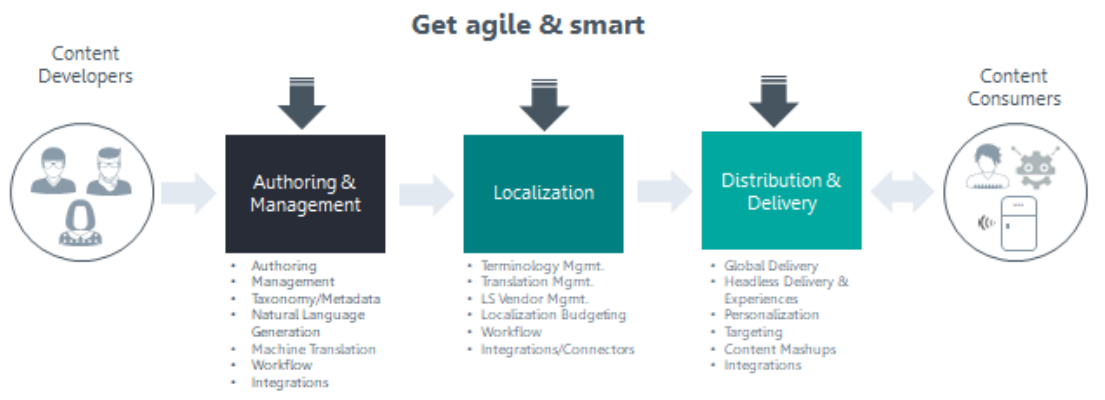

Fig. 3. Translation Automation shifting to Authoring and Management workflow

\section{From Content Management Systems to Content Intelligence Management Systems}

The content intelligence as seen in the previous chapter is derived during intelligent content development using metadata within localization processes supporting digital experience acceleration and multi-channel deliveries. Digital-first distribution being continuous, linear is no more good enough. Authoring, content management and localization can be agile and iterative. Commercial flexibility, such as monthly or quarterly utility billing, is paired with overlapping handoffs, smooth management of updates, and economical handling of bite-sized content. Besides new commercial models, this demands new translation technologies to unfragment traditional tech in favour of content lifecycle. The next-generation tech is agile. An agile content intelligence management system is designed from the ground up to support continuous localization, building an intelligent content supply chain with: 
- High levels of automation and visibility

- Deep integration with new hybrid CAT and linguistic AI tools (including neural machine translation)

- Content connectors and APIs that make it easy to embed translation into business workflows to connect a content repository to a localization management solution. Connector functionality ranges from the simple (e.g. automated logins to cloud file-sharing services) to the complex (e.g. embedding translation modules into an on-premises content management system).

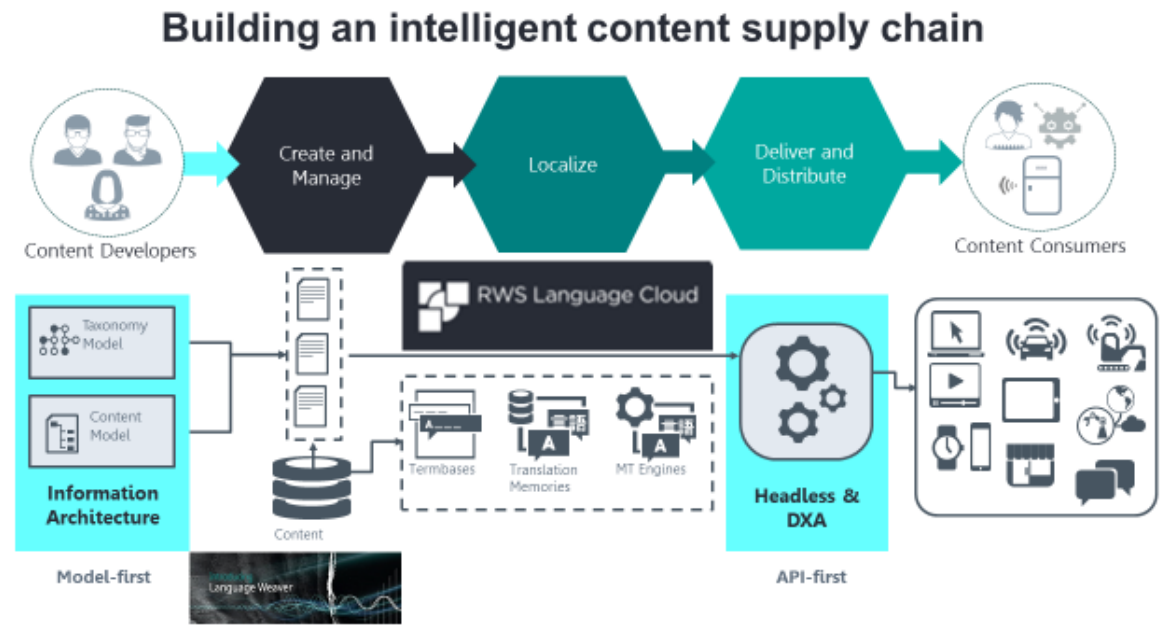

Fig. 4. Intelligent Content Supply chain

The key benefits are to go to market sooner, content connectors jump-start the content translation process so it is more agile, streamlined and efficient without the overhead of manual project standardized tasks less subject to error.

As projects get smaller and faster, technology must be used to eliminate overhead as much as possible by automating handoffs and repetitive tasks for each project. Continuous localization is the best model for managing large volumes of content and more iterations. To keep up with market demand for more personalized content, it's essential to invest in a robust technology framework built for continuous localization.

Continuous content creation requires dynamic, real-time localization, which is why continuous localization helps you achieve:

- Faster time to market: Translate content as needed.

- Higher quality translations: Minimize errors by reducing manual effort.

- Cost reduction: Eliminate repetitive project tasks.

- Process optimization: Analyze data for productivity gains. 


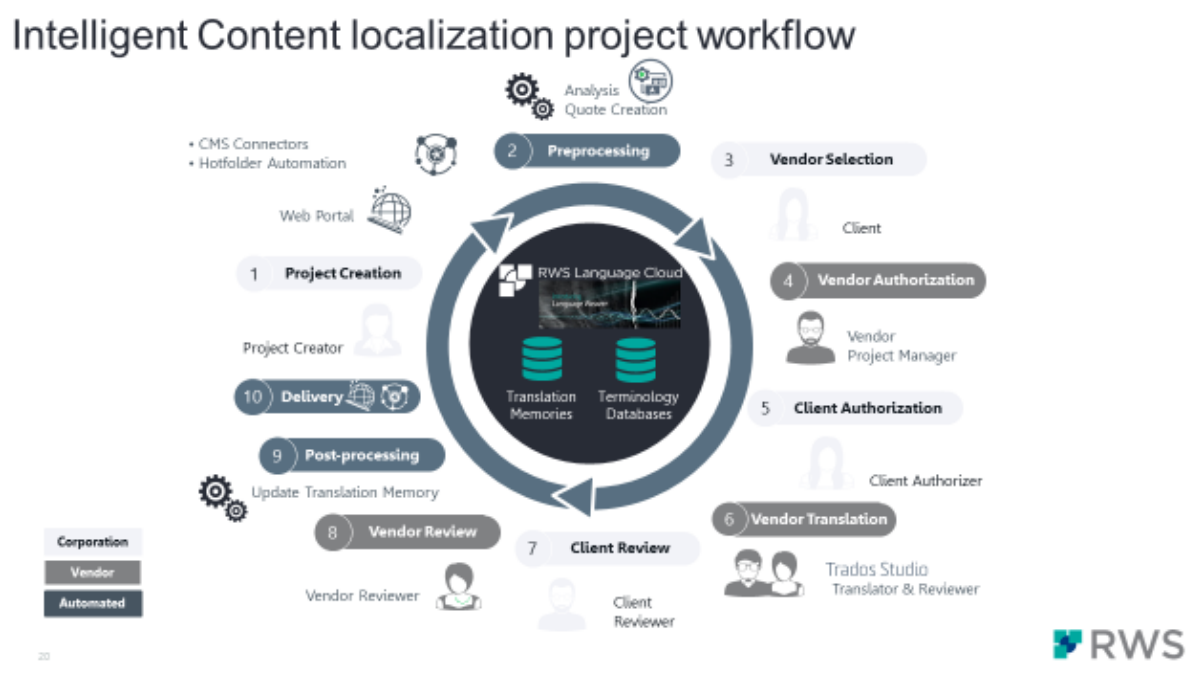

Fig. 5. Agile Intelligent Content localization management project workflow

Agile localization management is the use of specific technologies to manage translation as a continuous iterative process, rather than as a linear project. Content is translated as it is being developed.

An agile localization management system supports the following capabilities:

- Incremental flexibility

- Modern UX

- Easy file hand-off

- Side-by-side review

The key benefits include: Faster time to market, shorter turnaround time for greater automation and efficiency in end-to-end processes.

The solution constantly updates and evolves. Enabling staff around the world to detect and understand relevant information in other languages by sharing knowledge with one another. In a world without language barriers, this helps ensure local laws and regulations are being followed correctly worldwide.

Subject matter linguistic expertise is brought in to ensure the highest quality localizations, the longevity of the content through industry specific compliance standards, and helps Linguistic AI get smarter and smarter. 